Article

\title{
Expression Changes of MHC and Other Immune Genes in Frog Skin during Ontogeny
}

\author{
Quintin Lau ${ }^{1, *}$, Takeshi Igawa ${ }^{2}$, , Shohei Komaki ${ }^{3}$ and Yoko Satta ${ }^{1}$ \\ 1 Department of Evolutionary Studies of Biosystems, Sokendai (The Graduate University for Advanced \\ Studies), Kamiyamaguchi 1560-35, Hayama, Kanagawa 240-0193, Japan; satta@soken.ac.jp \\ 2 Amphibian Research Center, Hiroshima University, 1-3-1, Higashi-Hiroshima, Hiroshima 739-8526, Japan; \\ tigawa@hiroshima-u.ac.jp \\ 3 Division of Biomedical Information Analysis, Iwate Tohoku Medical Megabank Organization, Disaster \\ Reconstruction Center, Iwate Medical University, Idaidori 1-1-1, Yahaba, Iwate 028-3694, Japan; \\ komaki@iwate-med.ac.jp \\ * Correspondence: quintin@soken.ac.jp
}

Received: 3 December 2019; Accepted: 31 December 2019; Published: 6 January 2020

Simple Summary: Tadpoles undergo many changes in physiology and immunology until metamorphosis into adult frogs. Major histocompatibility complex (MHC) molecules are an important part of vertebrate adaptive immunity, and our study measured the expression of two MHC genes (MHC class I and II) in skin during six tadpole stages of the Montane Brown frog (Rana ornativentris). First, using a qPCR method, we found that both MHC class I and II expression significantly increased between stage 24/25 ('early') and stage 28 ('mid') tadpole skin. Then, we conducted next-generation sequencing for 'early', 'mid' and 'late' stage tadpole skin mRNA of both R. ornativentris and a model species, Xenopus tropicalis, and confirmed that MHC expression increased from the 'mid' stage. We also performed further analyses of transcriptome data and found that several immune-related gene ontology terms were upregulated from the 'mid' tadpole stage. Our findings probably support that both MHC class I and II have a functional role during tadpole development.

Abstract: Anuran amphibians undergo major physiological and immunological changes following metamorphosis. Genes of the major histocompatibility complex (MHC) code for receptors important for vertebrate adaptive immunity. We used qPCR to measure skin $M H C$ expression in six different ontological stages of Rana ornativentris ( $n=10$ per stage); normalized MHC class I and II expression at the mRNA level was significantly higher in stage 28 (mid-larval) compared to stages 24/25 (early-larval) tadpoles. Subsequent transcriptomic analyses of three tadpole (early-, mid-, and late-larval) stages of $R$. ornativentris and model species Xenopus tropicalis focused on mRNA expression of immune-related genes in the skin. Normalized expression of most MHC class I and II transcripts in both species were significantly higher in mid- and late-larval stages compared to early-larval stage. In addition, gene ontology $(\mathrm{GO})$ analyses of differentially expressed transcripts revealed several immune-related GO terms that were significantly upregulated from the mid-larval stage. Our study provides evidence that both MHC class I and II is expressed during development in both R. ornativentris and X. tropicalis.

Keywords: anuran development; major histocompatibility complex; Rana; Xenopus; transcriptome

\section{Introduction}

Anuran amphibians have a biphasic life cycle featuring a pre-metamorphic tadpole stage usually inhabiting fully aqueous environments, and a post-metamorphic adult stage inhabiting aqueous and/or terrestrial environments depending on the species. Based on model Xenopus frogs, 
tadpoles are considered to have a poor or immature adaptive immune system [1,2] as well as some components of innate immunity [3,4]. At metamorphosis, anurans are known to go through a complete physiological reorganization; this includes components of the immune system like expression of major histocompatibility complex (MHC) molecules (reviewed by Rollins-Smith, 1998) [5]. The MHC is an essential component of vertebrate adaptive immunity because $M H C$ genes code for membrane-bound glycoproteins that recognize, bind and present specific antigens to $\mathrm{T}$ lymphocytes. There are two major classes of MHC: MHC class I (MHC-I) molecules predominantly recognize and present endogenous antigenic peptides (e.g., from viruses) to cytotoxic T cells, while MHC class II (MHC-II) molecules present exogenous antigens (e.g., from fungi and bacteria) to helper $\mathrm{T}$ cells.

There have been reports of tadpole susceptibility to infectious agents like Perkinsea-like protists [6] and ranaviruses [7], as well as trematode-associated tadpole mortality [8]. Furthermore, a study demonstrated fatality in tadpoles after exposure to chytrid fungus Batrachochytrium dendrobatidis $(\mathrm{Bd})$, possibly due to toxins released by the fungus, as well as variation in susceptibility between species [9], although there is no concrete evidence that ties Bd to tadpole mortality. Further research into anuran immune expression and function during ontogeny is vital for broadening our understanding of host-pathogen dynamics, in lieu of the presence of various infectious pathogens that may affect tadpoles.

In general, the expression of MHC in anurans during ontogeny is not widely studied, and has been predominantly restricted to model organism Xenopus laevis. A study in X. laevis indicated that protein expression of MHC II is generally ubiquitous during ontogeny [10]; specifically, there is early expression in spleen, thymus and B cells, and expression in skin was detected after the mid-larval life stage. It appears that MHC class Ia (classical) expression is low during ontogeny based on northern blot analyses of thymus, spleen and skin mRNA [11,12]. However, a later study of lymphocytes in the spleen and thymus of X. laevis mid-stage tadpoles using fluorescence microscopy and flow cytometry supported MHC class I surface expression [13].

Since most studies of tadpole MHC expression have focused on Xenopus frogs, applying such findings to all anurans could be premature, considering they are among the most diverse vertebrates with at least 6981 species [14] and with the last common ancestor of Anura appearing around 210 million years ago $[15,16]$. Adding to this, there is some evidence that $M H C$ class I mRNA is expressed in tadpoles of Korean frogs [17]. In our previous transcriptome study of Japanese frogs, we found low normalized expression of both MHC class I and II transcripts in a few samples of Rana japonica and R. ornativentris mid-larval tadpoles (stage 28) [18]. Thus, in this study we wanted to expand on the knowledge related to MHC expression during anuran ontogeny, and conducted an in-depth quantification of mRNA expression levels of $M H C$ in a non-model amphibian species. We focused our examination to expression in skin, as it is a barrier between the host and the environment and is also the potential target site entry for some pathogens.

In this study, we quantified mRNA expression levels of $M H C$ class Ia and II during various stages of ontogeny of our selected study species, Rana ornativentris (Montane Brown frog) using quantitative PCR (qPCR). We subsequently used next-generation sequencing to establish transcriptome data of both $R$. ornativentris and Xenopus tropicalis tadpoles, and investigated expression of MHC and differential expression of other immune-related genes.

\section{Materials and Methods}

\subsection{Rana ornativentris Samples}

A proportion of two separate Rana ornativentris egg mass clusters were collected in January 2016 from Yokohama Nature Observation Forest Park, Kanagawa prefecture, Japan ( $35^{\circ} 20^{\prime} 22^{\prime \prime}$ N, 139 $35^{\prime} 15^{\prime \prime}$ E). Both egg clusters were collected within $72 \mathrm{~h}$ after fertilization, and were approved by Sokendai Research committee (approval number 46) and with official permission from the park. The two partial egg clusters (egg cluster A and B) were reared with $12 \mathrm{~h}: 12 \mathrm{~h}$ light:dark cycle in separate water containers ( $42 \mathrm{~cm}$ width $\times 58 \mathrm{~cm}$ length $\times 20 \mathrm{~cm}$ depth) containing $15-30 \mathrm{~L}$ of aged water with aeration, 
and water was replaced every 3-7 days. Once tadpoles reached feeding stage (after stage 24), they were fed daily with Pleco Spirulina Wafers (Tetra, Melle, Germany).

Animals ( $n=5$ per stage per egg cluster) were collected at six life/ontological stages (Figure 1, Table S1) according to those described in the closely related Rana japonica [19,20]: (i) stage 20-appearance of external gills, (ii) stage 24/25 - external gills covered and appearance of hindlimb buds, (iii) stage 28/29-hindlimb bud is 1.5-2.0 fold of its diameter, (iv) stage 33-appearance of prehallux, (v) stage 37-protrusion of one or both forelimbs and (vi) stage 40-full disappearance of tail. A total of 60 tadpoles were collected for RNA extraction and MHC expression analyses (two egg clusters, six life stages and five animals per stage).

Animals were euthanized by immersion in tricaine methanesulfonate (MS222, 0.5-3 g/L water). Next, skin was immediately dissected for RNA extraction and the remaining body was preserved in RNAlater solution (Applied Biosystems, Carlsbad, CA, USA) at $-80^{\circ} \mathrm{C}$. Dissected skin samples were immediately homogenized in ISOGEN (Nippon Gene, Tokyo, Japan) and stored at $-80^{\circ} \mathrm{C}$ for less than one month before RNA extraction following manufacturer's protocol. RNA was then synthesized into first-strand complementary DNA (cDNA) using PrimeScript ${ }^{\mathrm{TM}} \mathrm{RT}$ reagent kit (Takara Bio Inc., Otsu, Japan).

Additional RNA samples, included for investigating MHC expression, were retrospectively extracted from tissues preserved in RNAlater solution using the same isolation procedure and synthesized into cDNA. This included (a) whole bodies of stage $24 / 25$ tadpoles ( $n=5$ animals per cluster) to compare $M H C$ expression levels to that of skin in the same stage, and (b) ventral skin ( $n=6$ total) and spleen ( $n=6$ total) of adult frogs collected from Hiroshima prefecture and used in our previous studies $[18,21,22]$.

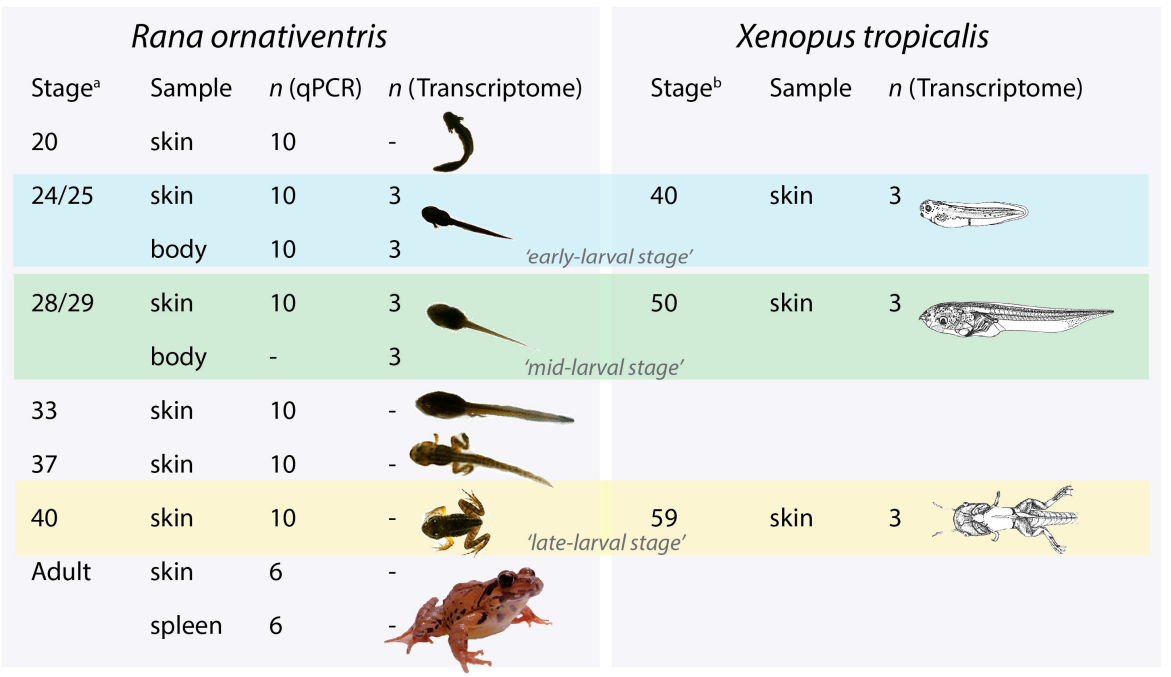

Figure 1. Overview of Rana ornativentris and Xenopus tropicalis samples used for qPCR and transcriptome analyses in this study. ${ }^{a} R$. ornativentris stage approximations based on Tahara $[19,20] .{ }^{b} X$. tropicalis stage approximations based on Nieuwkoop and Faber [23]. Full stage approximations in Table S1. Images of frogs in all figures are not to scale (source: Q. Lau for R. ornativentris, Nieuwkoop and Faber (1994) via Xenbase for X. tropicalis).

\subsection{Primer Design and Generation of Standards for Quantitative PCR ( $q P C R$ )}

We previously generated transcriptome data from a single $R$. ornativentris adult individual and additionally used molecular cloning in seven individuals to characterize $780 \mathrm{bp}$ and $754 \mathrm{bp}$ sequences for $M H C$ class I a (classical) and $M H C$ class II beta chain, respectively [18,21]. In the present study, we developed MHC class I and II primers for quantitative polymerase chain reaction (qPCR); we independently aligned all previously characterized MHC class I and II variants [18,21], and designed primers in conserved regions that amplified across exon boundaries (Table 1). Primers 
were designed based on variants from one or more $M H C$ class I and class II loci (3-5 MHC class I and 1-2 MHC class II variants per individual were identified previously), thus our primers may detect all expressed MHC class I and II loci in the species. Primers for reference gene glyceraldehyde 3-phosphate dehydrogenase (GAPDH, Table 1) were also developed based on conserved regions of assembled contigs from transcriptome data [18] of $R$. ornativentris aligned with that of related species $R$. japonica and $R$. tagoi tagoi (GenBank accession numbers MK541925-MK541927).

Table 1. Summary of primers used for qPCR in R. ornativentris.

\begin{tabular}{cccccc}
\hline Target Gene & Target Species & Boundary Crossed & Forward Primer $\left(\mathbf{5}^{\prime}-\mathbf{3}^{\prime}\right)$ & Reverse Primer $\left(\mathbf{5}^{\prime}-\mathbf{3}^{\prime}\right)$ & Amplicon Length $\mathbf{( b p )}$ \\
\hline MHC class I & R. ornativentris & exon $3-4$ & TCCCGACCATGAATGAGG & GACTGACGATGACCCCACA & 190 \\
MHC class II beta & R. ornativentris & exon $3-4$ & CACAGCAGCCTGGAGACA & AGCACAAATCCCACAATCC & 98 \\
GAPDH & Japanese Rana & exon 5-6 & CCAACGTGTCTGTGGTTGAC & TCCCAGAATTCCCTTCAGTG & 113 \\
\hline
\end{tabular}

To generate standards for each of the three genes, we utilized plasmid DNA generated by traditional PCR and molecular cloning. Traditional PCR amplification was conducted using Applied Biosystems ${ }^{\circledR}$ VeritiC thermal cycler in $10 \mu \mathrm{L}$ reactions containing approximately $100 \mathrm{ng}$ of cDNA (from skin of a stage 40 R. ornativentris tadpole), $0.25 \mathrm{U}$ TaKaRa Ex Taq (Takara Bio Inc.), 1X Ex Taq buffer, $0.5 \mathrm{mM}$ each dNTP and $0.5 \mu \mathrm{M}$ each primer. Cycle condition comprised of 30 cycles of $30-\mathrm{s}$ denaturation at $98{ }^{\circ} \mathrm{C}, 30$-s annealing at $58{ }^{\circ} \mathrm{C}$, and 30 -s extension at $72{ }^{\circ} \mathrm{C}$, followed by a final extension of $72{ }^{\circ} \mathrm{C}$ for $3 \mathrm{~min}$. Sequences of PCR products were confirmed by sequencing: in brief, they were purified with ExoSAP-IT ${ }^{\circledR}$ (Affymetrix Inc., Santa Clara, CA, USA) and sequenced with BigDye ${ }^{\circledR}$ Terminator Cycle Sequencing Kit (Applied Biosystems, Foster City, CA, USA) and ABI 3130xl automated sequencer. For cloning, PCR products were ligated using T-Vector pMD20 and DNA Ligation Kit 2.0 (Takara Bio Inc.) and incubated for $30 \mathrm{~min}$ at $16^{\circ} \mathrm{C}$. Next, ligated reactions were transformed into JM 109 Competent Cells (Takara Bio Inc.) following manufacturer instructions. A single positive clone was cultured overnight in $5 \mathrm{~mL}$ of LB broth containing $100 \mu \mathrm{g} / \mathrm{mL}$ ampicillin, and plasmid DNA was purified using QIAprep ${ }^{\circledR}$ Spin Miniprep kit (Qiagen, Hilden, Germany) following manufacturer instructions. From plasmid DNA, we prepared aliquots of standards by 1:10 serial dilutions $(10 \mathrm{pg} / \mu \mathrm{L}$, $1 \mathrm{pg} / \mu \mathrm{L}, 0.1 \mathrm{pg} / \mu \mathrm{L}, 10 \mathrm{fg} / \mu \mathrm{L}$ and $1 \mathrm{fg} / \mu \mathrm{L})$.

\subsection{MHC Expression in R. ornativentris Using Quantitative PCR (qPCR)}

We used qPCR to measure expression levels for the two test genes (MHC class I and class II) and the reference gene $(G A P D H)$ from the 60 R. ornativentris tadpole skin samples and additional body and adult samples. Using Thermal Cycler Dice ${ }^{\circledR}$ Real Time System II (Takara), qPCR was conducted in $10 \mu \mathrm{L}$ triplicate reactions containing $2 \mu \mathrm{L}$ of template (either standard or sample DNA), 1X SYBR Premix Ex Taq II (Tli RNaseH Plus; Takara Bio Inc.) and $0.8 \mu \mathrm{M}$ each primer. Reaction conditions were (i) initial denaturation stage: $95^{\circ} \mathrm{C}$ for 30-s; (ii) PCR stage: 40 cycles of $95{ }^{\circ} \mathrm{C}$ for 5 -s and $55^{\circ} \mathrm{C}$ for 60 -s; (iii) dissociation stage: $95^{\circ} \mathrm{C}$ for 15 -s then $60{ }^{\circ} \mathrm{C}$ for 30 -s then $95^{\circ} \mathrm{C}$ for 15 -s. Quantity was calculated using standard curves and averages from triplicate reactions. Relative expression of either $M H C$ class I or II was determined by ratio of quantity divided by that of GAPDH.

\subsection{Statistical Analyses of qPCR Expression Data}

All statistical analyses were conducted using R v 3.3.2. All data were log-transformed after quantile-quantile plots and Shapiro-Wilk normality tests confirmed non-normal distribution (data not shown). One-way analysis of variance (ANOVA) was used to compare $M H C$ expression between the six tadpole stages, using egg cluster as a block (model: log- $M H C$ normalized expression life stage + egg cluster). Subsequently, we used ANOVA to compare MHC expression in stage 24/25 tadpole skin with whole body. We also compared $M H C$ expression between adult skin and spleen samples to validate our previous expression data extrapolated from transcriptome data, whereby we identified higher normalized expression of $M H C$ in spleen relative to skin [18]. We used $p<0.05$ to denote statistical significance. 


\subsection{Transcriptome Analyses of Immune Expression in R. ornativentris Tadpoles}

To validate the changes of MHC class I and II expression during ontogeny of $R$. ornativentris tadpoles detected by qPCR and investigate changes in overall immune gene expression, we subsequently conducted transcriptome analyses in a subset of samples: 'early-larval' stage 24/25 skin $(n=3)$ and whole body $(n=3)$, 'mid-larval' stage $28 / 29$ skin $(n=3)$ and whole body $(n=3)$ and 'late-larval' stage 40 skin $(n=3)$. The same RNA samples $(2-10 \mu \mathrm{g}$ of RNA per sample) extracted for qPCR were also used for library construction using NEBNext Ultra Directional RNA Library Prep Kit for Illumina ${ }^{\circledR}$ (New England Bio Labs, MA, USA). Short DNA sequencing (PE150 reads) was conducted at Novogene Co. Ltd. (Beijing, China) using cDNA Illumina HiSeq2000 sequencing system (3 GB per sample). Sequenced reads were filtered by removing reads containing adapters, $\mathrm{N}>10 \%$, or low quality (Qscore $\leq 5$; Novogene Co. Ltd.). De novo assembly was then conducted with default settings in Trinity version 2.8.3 [24] using combined reads from the R. ornativentris libraries to create a reference data set.

Reads from each sample were aligned back to the reference transcript data set using kallisto (v0.45.1) [25] and transcript abundances were reported as transcripts per million (TPM). We isolated MHC class I and II transcripts from the libraries using NCBI-BLAST-2.2.31+ with E-values $\leq 1$ $\times 10^{-5}$ for blastn search with previously characterized $R$. ornativentris MHC sequences $[18,21]$ as queries, and extrapolated TPM values. We conducted differential gene analyses to test for significant differences in MHC expression and extrapolate other immune-related transcripts with significant expression differences between larval stages (early vs. mid vs. late) or between tissues (skin vs. body). Differentially expressed transcripts (including MHC) between stages or tissues were determined using likelihood ratio test (LRT) implemented in edgeR (3.9) [26]. Pairwise comparisons between tadpole stages or tissues were conducted using false discovery rate (FDR) cut-off of 0.05 , and transcripts with $\log$-2-fold-changes $\log \mathrm{FC}>|2|$ were extracted. All differentially expressed (DE) transcripts were collated and annotated with NCBI-BLAST-2.2.31+ with E-values $\leq 1 \times 10^{-5}$ against Xenopus tropicalis 9.1 protein dataset from Xenbase [27] (http://www.xenbase.org/, RRID:SCR_003280). We then submitted the blast results, in the form of GenBank and RefSeq IDs, for gene ontology (GO) enrichment analyses implemented in DAVID 6.8 [28]. All enriched GO terms related to the immune system were collected using a threshold of gene count $\geq 3$ and $p<0.1$.

\subsection{MHC and Immune Gene Expression in Xenopus Tadpoles}

Based on MHC expression data from Rana ornativentris, we were interested to explore MHC class expression in Xenopus skin mRNA at pre-metamorphic stages. Thus, we also conducted transcriptome analyses of Xenopus tropicalis tadpole skin during ontogeny. We collected X. tropicalis skin samples from three tadpole stages ( $n=3$ individuals per stage), similar to stages used for $R$. ornativentris. The three stages are stage 40 ('early-larval'), 50 ('mid-larval') and 59 ('late-larval') based on X. laevis [23], which is equivalent to around stages $21 / 22,27$ and 37 , respectively, in $R$. ornativentris, following inter-species stage comparisons $[19,20,29,30]$ (Table S1). All individuals were euthanized in a similar approach (see above) and stored in RNAlater solution at $-80{ }^{\circ} \mathrm{C}$ until RNA extraction and cDNA synthesis, library construction and Illumina ${ }^{\circledR}$ sequencing using the same procedures as in $R$. ornativentris. Using kallisto, clean reads were mapped onto the X. tropicalis genome (v9.1 genome assembly), which was downloaded from Xenbase [27]. Differential expression analyses between stages using LRT as well as GO enrichment analyses followed the same procedures as in R. ornativentris, while MHC expression levels were extrapolated based on transcripts mapped to $M H C$ genes already annotated in the genome.

\section{Results and Discussion}

\subsection{MHC Expression in R. ornativentris Using qPCR Analyses}

Among non-model amphibians, this is one of the first studies to quantify changes in MHC expression during ontogeny. mRNA expression of $M H C$ class $I$ in $R$. ornativentris tadpole skin 
was low during early developmental stages (stage 20, and stage 24/25), and significantly increased in all subsequent stages from stage 28/29 ( $p<0.00001$, Figure 2A). In a similar pattern to MHC-I, mRNA expression of MHC-II was significantly lower in stages 20 and 24/25 compared to mid- and late-developmental stages ( $p<0.00001$, Figure 2B). Throughout all developmental stages studied, overall expression of $M H C-I I$ was about 10-fold lower than that of MHC-I (Table S2).
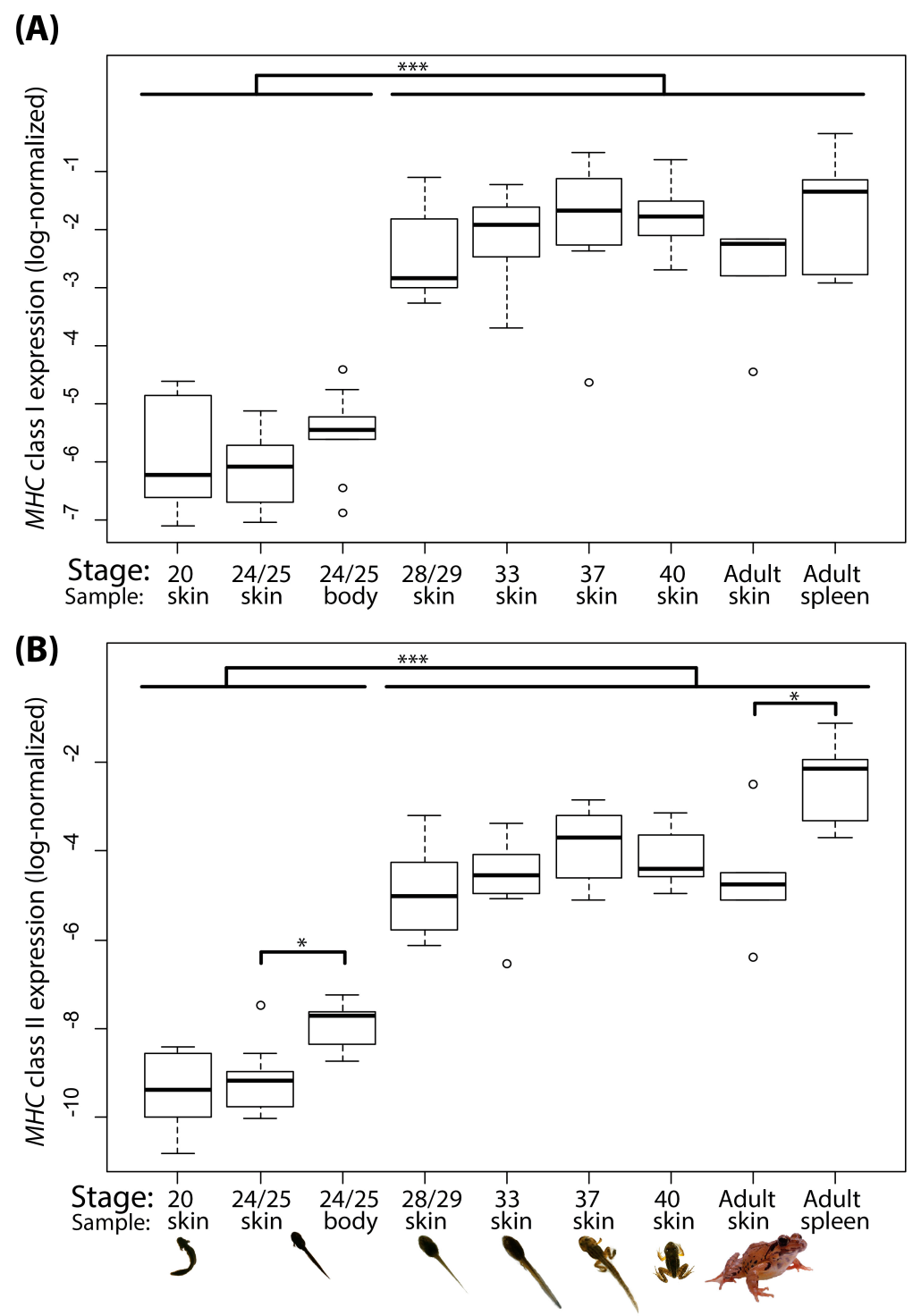

Figure 2. Boxplot of (A) MHC class I and (B) MHC class II expression in mRNA of Rana ornativentris tadpole skin during six ontological stages and other samples: stage $24 / 25$ body, and adult skin and spleen. MHC expression values from qPCR were normalized by the GAPDH control gene and log-transformed for normality (log-normalized). ${ }^{*} p<0.05,{ }^{* * *} p<0.0001$.

Next, we checked whether the low MHC class I and II expression in the skin of stage 24/25 tadpoles was also low in the 'whole body' samples of the same individuals. We found that expression was slightly higher in whole body of tadpoles than the skin, but this was statistically significant only for MHC-II (MHC-I: $p=0.0567$, MHC-II: $p<0.001$, Figure 2). We also compared expression between spleen and skin from $R$. ornativentris adults $(n=6)$ : MHC-I expression was not significantly different ( $p=0.054$, Figure 2A) since it is ubiquitously expressed in most mature cells, whereas MHC-II was significantly higher in spleen than skin $(p=0.022$, Figure $2 \mathrm{~B})$. These qPCR results were consistent with 
the normalized expression levels determined from transcriptome data of a single individual in our previous study [18].

\subsection{MHC Expression in R. ornativentris and X. tropicalis Using Transcriptome Analyses}

To validate our findings of higher $M H C$ expression in tadpole skin during later stages of ontology from qPCR analyses, we prepared transcriptomic data for three developmental stages of both $R$. ornativentris and $X$. tropicalis (early-, mid- and late-larval stages, with approximately similar stages between the two species, see Table S1). In R. ornativentris, we found two MHC-I and one $M H C-I I$ transcript with higher expression in mid- and late- compared to early-larval stage skin (Table 2); however, this was significant only in the $M H C-I I(\mathrm{M}>\mathrm{E}$ : $\log \mathrm{FC}=3.81)$ and a single $M H C-I(\mathrm{M}>$ $\mathrm{E}: \log \mathrm{FC}=2.51 ; \mathrm{L}>\mathrm{E}: \log \mathrm{FC}=5.38)$ transcript using a strict corrected cut-off of $\mathrm{FDR}<0.05$. Our skin-related normalized expression data is comparable to our qPCR results, whereby significantly higher MHC class I and II expression in skin was detected at stage 28/29 (mid-larval). However, there were no significant differences between body and skin in $M H C$ normalized expression levels based on transcriptome data (Table 2), despite a significant difference in MHC-II detected using qPCR (Figure 2B). This may be attributed to differences in normalization and statistical approaches between the two different methods. Nevertheless, there is opportunity in the future to explore MHC expression (gene and protein level) in other specific tissues during tadpole development.

Table 2. Normalized expression of $M H C$ class I and II transcripts between different larval stages (E-early, M-mid and L-late, see Table S1) or tissues of Rana ornativentris (Raor) and Xenopus tropicalis (Xetr), represented by TPM (transcripts per million) values, and logFC and false discovery rate (FDR) values of pairwise comparisons between samples. Transcripts with significantly different normalized MHC expression levels (FDR < 0.05) are indicated in bold.

\begin{tabular}{|c|c|c|c|c|c|c|}
\hline $\begin{array}{l}\text { Sample or } \\
\text { Comparison }\end{array}$ & $\begin{array}{l}\text { Raor MHC-I } \\
\text { (Transcript 1) }\end{array}$ & $\begin{array}{l}\text { Raor MHC-I } \\
\text { (Transcript 2) }\end{array}$ & $\begin{array}{c}\text { Raor } \\
\text { MHC-II }\end{array}$ & $\begin{array}{c}\text { Xetr MHC-I } \\
\text { [hla-a] } \\
\text { (NP_001106536.1) }\end{array}$ & $\begin{array}{c}\text { Xetr MHC-II } \\
\text { [hla-dra] } \\
\text { (XP_017951884.1) }\end{array}$ & $\begin{array}{c}\text { Xetr MHC-II } \\
\text { [hla-drb1] } \\
\text { (NP_001039259.1) }\end{array}$ \\
\hline \multicolumn{7}{|l|}{$T P M$ mean $\pm S D$} \\
\hline E_body & $1.9 \pm 1.4$ & $7.7 \pm 3.2$ & $0.0 \pm 0.0$ & - & - & - \\
\hline M_body & $18.1 \pm 3.4$ & $19.4 \pm 8.6$ & $3.1 \pm 2.3$ & - & - & - \\
\hline E_skin & $1.9 \pm 0.8$ & $6.7 \pm 5.5$ & $0.2 \pm 0.2$ & $2.0 \pm 2.6$ & $3.0 \pm 2.6$ & $43.0 \pm 5.6$ \\
\hline M_skin & $25.3 \pm 2.7$ & $50.0 \pm 21.2$ & $7.5 \pm 4.2$ & $484.3 \pm 184.8$ & $741.4 \pm 354.3$ & $1957.7 \pm 877.9$ \\
\hline L_skin & $10.8 \pm 6.6$ & $41.1 \pm 4.9$ & $10.0 \pm 7.7$ & $608.5 \pm 290.6$ & $711.9 \pm 877.9$ & $2374.0 \pm 1492.0$ \\
\hline \multicolumn{7}{|l|}{$\log F C(F D R)$} \\
\hline skin: E v M & $3.81(0.008)$ & $3.00(0.103)$ & $2.51(0.024)$ & $8.27\left(1.50 \times 10^{-24}\right)$ & $8.33\left(4.58 \times 10^{-27}\right)$ & $5.93\left(4.15 \times 10^{-21}\right)$ \\
\hline skin: E v L & $2.37(0.400)$ & $2.55(0.297)$ & $5.38(0.037)$ & $8.73\left(5.57 \times 10^{-47}\right)$ & $8.33\left(3.92 \times 10^{-43}\right)$ & $6.30\left(1.87 \times 10^{-40}\right)$ \\
\hline skin: M v L & $-1.40(0.550)$ & $-0.46(1)$ & $0.27(1)$ & $0.49(1)$ & $0.04(1)$ & $0.39(1)$ \\
\hline body v skin: E & $-0.02(1)$ & $-0.29(1)$ & $4.70(1)$ & - & - & - \\
\hline body v skin: $\mathrm{M}$ & $0.63(0.904)$ & $1.54(0.465)$ & $1.39(0.682)$ & - & - & - \\
\hline body v skin: E/M & $0.58(1)$ & $1.16(0.751)$ & $1.46(0.922)$ & - & - & - \\
\hline
\end{tabular}

To investigate species differences in $M H C$ expression patterns in developmental stages, we also conducted transcriptome analyses in Xenopus tadpole skin. In X. tropicalis, we found one MHC-I and two MHC-II transcripts (corresponding to the MHC class II alpha and beta chain) with significantly higher normalized expression in mid- and late- versus early-larval stage skin $(\log \mathrm{FC}=5.93-8.73$, FDR < 1E-20). The low MHC expression in early-larval and higher expression at mid- and late-larval stage skin was also validated using preliminary standard PCR amplification in both X. tropicalis and X. laevis (Supplementary Methods, Figure S1).

Our results provide supporting evidence of $M H C$ class I expression in X. laevis skin mRNA during development using sensitive detection approaches (PCR and especially next-generation sequencing (NGS)). This investigation of MHC expression in Xenopus tadpoles using NGS approaches provides a springboard for future studies to quantify this expression at a more in-depth level and re-assess expression differences across additional tissues and developmental stages. 


\subsection{Differential Expression of Immune-Related Genes in R. ornativentris and X. tropicalis Tadpoles}

In pairwise comparisons of skin samples at different stages (early-, mid- and late-larval stages), we found 740-2008 differentially expressed transcripts in R. ornativentris and 79-2708 in X. tropicalis (Table S3). When these transcripts were submitted for GO analyses in DAVID, we found that the wide range of differentially expressed gene numbers from pairwise comparisons were mainly due to the marked differences in cellular, developmental, neural and immunological processes between early- and late-larval stages of both species (Table S4). When focusing on the 'biological processes' parent term, we found 46-402 GO terms in R. ornativentris and 28-434 GO terms in X. tropicalis that were enriched at specific stages (Table S3). Among these GO terms, many immune-related terms were enriched in midand late-larval stage (24 and 4 terms in R. ornativentris, 48 and 38 terms in X. tropicalis, respectively) compared to early-larval stage tadpole skin (listed in Figure 3, Tables S5 and S6); one term (GO:0019882 antigen processing and presentation), which might be related to MHC function, was enriched in both species. In contrast, only non-immune-related GO terms were enriched in the early-larval stage tadpole skin compared to the latter two stages, including GO:0032501 single-multicellular organism process and GO:0007399 nervous system development (Table S4).

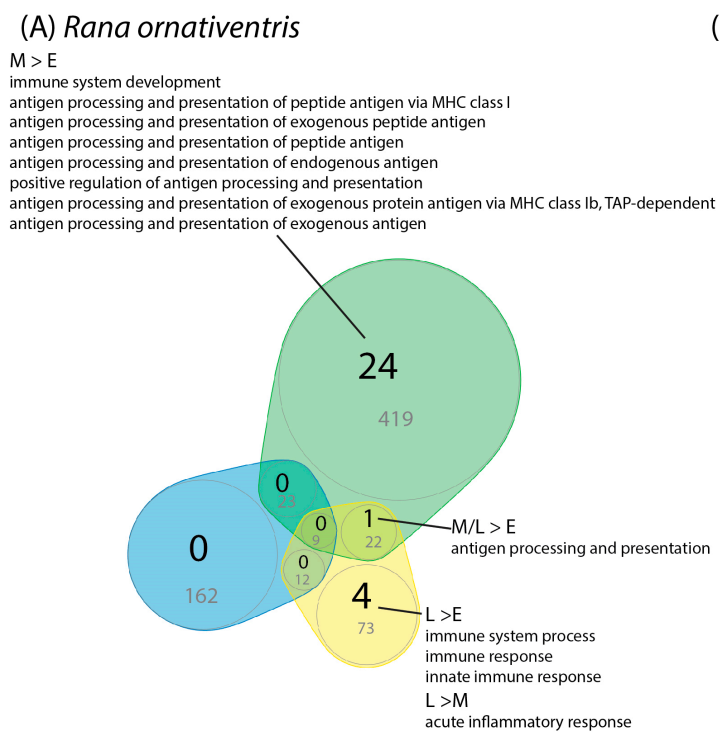

(B) Xenopus tropicalis
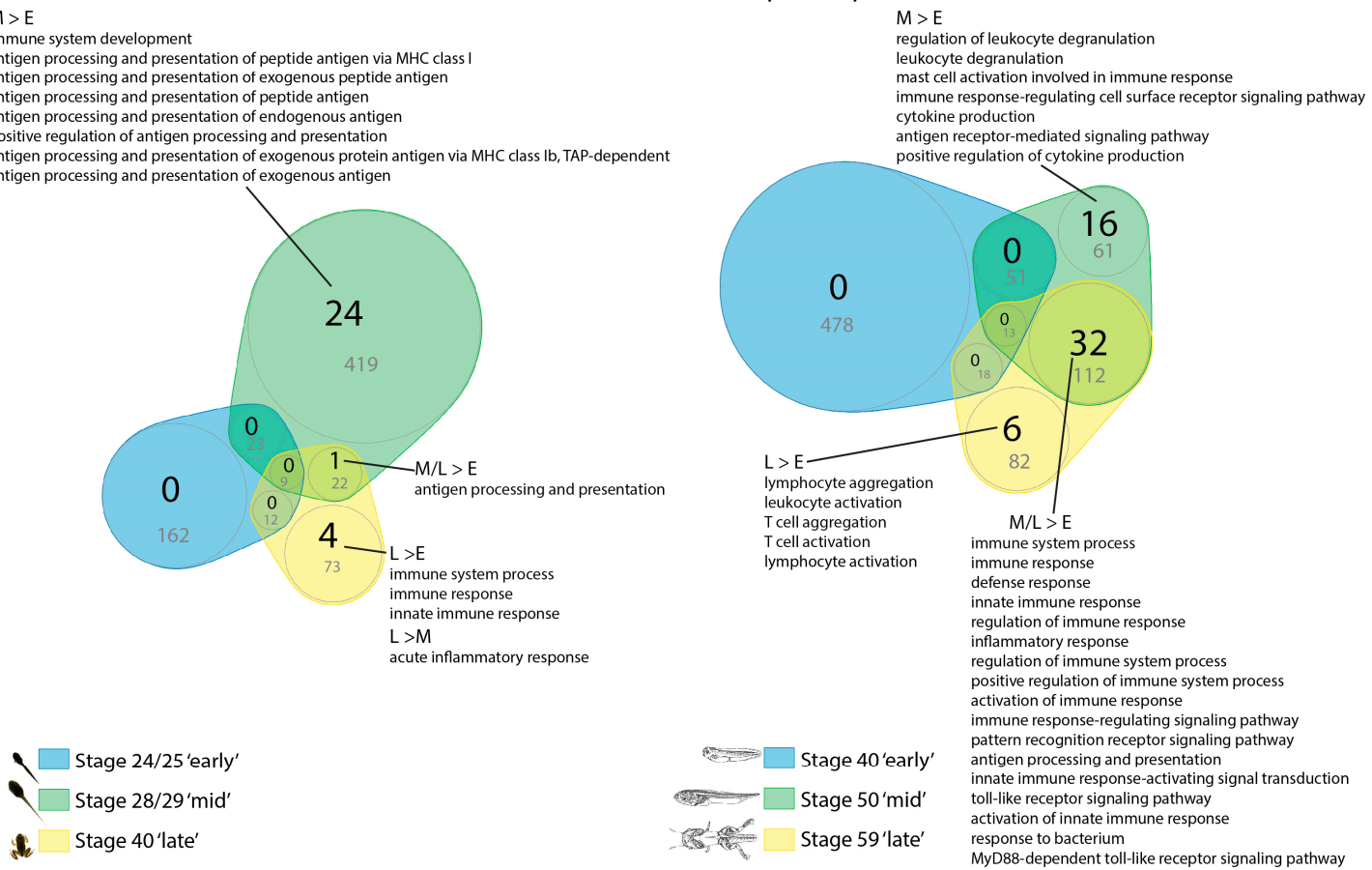

response to bacterium

Figure 3. Venn diagrams of gene ontology (GO) terms enriched in skin of early- (E), mid- (M) and late(L) larval stages from (A) Rana ornativentris and (B) Xenopus tropicalis. Number of immune-related enriched GO terms are in black (full list in Tables S5 and S6), and total number of enriched GO terms belonging to the 'biological process' parent category are in grey and also represented by circle sizes. Examples of enriched immune-related GO terms are listed in comparisons between stages (full list in Tables S5 and S6).

In $R$. ornativentris, $23 \mathrm{GO}$ terms related to antigen processing and presentation (including GO:0002474 antigen processing and presentation of peptide antigen via MHC class I) were enriched in mid-larval skin compared to early-larval skin (Figure 3A, Table S5). This adds further support to the significantly increased $M H C$ expression, which we detected using both qPCR and transcriptome approaches, especially for class I (Figure 2, Table 2). In X. tropicalis, GO:0019882 antigen processing and presentation, GO:0045087 innate immune response, and GO:0002224 toll-like receptor signaling pathway were among the numerous immune-related GO terms significantly enriched in mid- and 
late-larval skin compared to early-larval skin (Figure 3B, Table S6). However, no GO terms with specific mention of 'MHC' were enriched in this species.

The significant enrichment of immune-related genes from the mid-larval stage, concurrent with significant increases in $M H C$ expression, supports that marked development of the tadpole immune system occurs before stage 28 in R. ornativentris and stage 50 in X. tropicalis. There was only a single immune-related GO term enriched in late-larval versus mid-larval skin of $R$. ornativentris (GO:0002526 acute inflammatory response; Table S4). Similarly, no immune-related GO terms in skin were significantly enriched after the mid-larval stage of $X$. tropicalis; this suggests that further changes in the immune system do not occur during the remainder of X. tropicalis and R. ornativentris tadpole development, at least until after metamorphosis. There may also be some other factors that contribute to differences in developmental immunity between the two species: X. tropicalis might rely more on the innate rather than adaptive immune system, $R$. ornativentris has a higher number of $M H C$ loci (at least three MHC-I loci [21]) compared to Xenopus (single MHC-I locus [31]), and there are species differences in post-metamorphosed life-cycles (semi-aquatic versus fully aquatic). Nevertheless, high-coverage whole genome sequences for $R$. ornativentris would be required for a more comprehensive comparison between species.

Our transcriptomic study focused predominantly on skin samples, although we also examined some 'whole body' samples of $R$. ornativentris for a preliminary insight into tissue-specific differences. In comparisons within the same stages of early- and/or mid-larval stage $R$. ornativentris tadpoles, there were more differentially expressed transcripts and enriched GO terms in the body compared to skin (Table S3). Most of these enriched terms were not immune-related (Table S4), except GO:0006955 immune response, which was enriched in body compared to skin of combined early- and mid-larval stages (Table S5). Even so, we have established a foundation and database for further investigation of immune-gene expression in different specific tissues during larval development as well as immune responses to infectious diseases like ranaviruses and $\mathrm{Bd}$.

\section{Conclusions}

We conducted an in-depth analysis of $M H C$ class I and II expression in skin of a Japanese frog species during ontogeny using $\mathrm{qPCR}$ and next-generation sequencing approaches. We found that expression of $M H C$ at the mRNA level was low during early developmental stages, and significantly increased during and following the mid-larval stage. Future immunological assays will be important for validating the functional role of both MHC class I and II during ontogeny in anuran frogs. Analyses of skin transcripts also identified many immune-related GO terms that were upregulated from the mid-larval stage. Our additional examination in Xenopus frogs also supports an increased expression of $M H C$ and other immune-related genes from the mid-larval stage. Despite the distant evolutionary relationship between Xenopus and Rana spp., our congruent findings in both support that anuran species have $M H C$ expression in the skin during development.

Supplementary Materials: The following are available online at http://www.mdpi.com/2076-2615/10/1/91/s1, Supplementary Materials: Preliminary PCR of MHC class I and II in Xenopus frogs, Table S1: Approximation of tadpole stages, Table S2: Normalized (and log-normalized) MHC class I and II expression values of Rana ornativentris tadpole skin samples and other tissues, Table S3: Number of differentially expressed transcripts in pairwise comparisons of samples in Rana ornativentris or Xenopus tropicalis, Table S4: Top 10 enriched GO terms in each pairwise comparison of Rana ornativentris or Xenopus tropicalis tadpole samples, Table S5: Immune-related GO terms enriched in specific stages or tissues of Rana ornativentris tadpoles, Table S6: Immune-related GO terms enriched in skin of mid and/or late (M/L) compared to early (E) larval stages of Xenopus tropicalis tadpoles, Figure S1: Gel electrophoresis following PCR amplification of GAPDH, MHC class I, and MHC class II from early-, mid-, and late-larval stages of Xenopus laevis and X. tropicalis tadpole skin cDNA samples.

Author Contributions: Conceptualization, Q.L., T.I. and Y.S.; methodology, Q.L. and S.K.; analysis, Q.L., T.I. and S.K.; writing—original draft preparation, Q.L.; writing—review and editing, S.K., T.I. and Y.S.; supervision, Y.S.; funding acquisition, Q.L. All authors have read and agreed to the published version of the manuscript.

Funding: This work was supported by Grants-in-Aid for Scientific Research (KAKENHI) grant number 17K15053 to QL. 
Acknowledgments: We thank the staff at the Yokohama Nature Observation Forest Park and volunteers (especially Masaru Shinozuka) for permission and help with sampling of $R$. ornativentris egg clusters. We also thank Akira Sasaki for assistance with statistical analyses. Xenopus tropicalis and X. laevis were provided by the Amphibian Research Center (Hiroshima University) through AMED under Grant Number JP18km0210085.

Conflicts of Interest: The authors declare no conflict of interest.

\section{References}

1. Robert, J.; Ohta, Y. Comparative and developmental study of the immune system in Xenopus. Dev. Dyn. 2009, 238, 1249-1270. [CrossRef]

2. Du Pasquier, L.; Schwager, J.; Flajnik, M.F. The immune system of Xenopus. Annu. Rev. Immunol. 1989, 7, 251-275. [CrossRef] [PubMed]

3. De Jesús Andino, F.; Chen, G.; Li, Z.; Grayfer, L.; Robert, J. Susceptibility of Xenopus laevis tadpoles to infection by the ranavirus Frog-Virus 3 correlates with a reduced and delayed innate immune response in comparison with adult frogs. Virology 2012, 432, 435-443. [CrossRef] [PubMed]

4. Wendel, E.S.; Yaparla, A.; Melnyk, M.L.S.; Koubourli, D.V.; Grayfer, L. Amphibian (Xenopus laevis) tadpoles and adult frogs differ in their use of expanded repertoires of type I and type III interferon cytokines. Viruses 2018, 10, 372. [CrossRef] [PubMed]

5. Rollins-Smith, L.A. Metamorphosis and the amphibian immune system. Immunol. Rev. 1998, 166, $221-230$. [CrossRef] [PubMed]

6. Chambouvet, A.; Gower, D.J.; Jirků, M.; Yabsley, M.J.; Davis, A.K.; Leonard, G.; Maguire, F.; Doherty-Bone, T.M.; Bittencourt-Silva, G.B.; Wilkinson, M.; et al. Cryptic infection of a broad taxonomic and geographic diversity of tadpoles by Perkinsea protists. Proc. Natl. Acad. Sci. USA 2015, 112, E4743-E4751. [CrossRef]

7. Haislip, N.A.; Gray, M.J.; Hoverman, J.T.; Miller, D.L. Development and disease: How susceptibility to an emerging pathogen changes through anuran development. PLoS ONE 2011, 6, e22307. [CrossRef]

8. Goren, L.; Routtu, J.; Ben-Ami, F. Trematode-associated morbidity and mortality of tadpoles in Israel. Parasitol. Res. 2014, 113, 3833-3841. [CrossRef]

9. Blaustein, A.R.; Romansic, J.M.; Scheessele, E.A.; Han, B.A.; Pessier, A.P.; Longcore, J.E. Interspecific variation in susceptibility of frog tadpoles to the pathogenic fungus Batrachochytrium dendrobatidis. Conserv. Biol. 2005, 19, 1460-1468. [CrossRef]

10. Du Pasquier, L.; Flajnik, M.F. Expression of MHC class II antigens during Xenopus development. Dev. Immunol. 1990, 1, 85-95. [CrossRef]

11. Flajnik, M.F.; Kaufman, J.F.; Hsu, E.; Manes, M.; Parisot, R.; Du Pasquier, L. Major histocompatibility complex-encoded class I molecules are absent in immunologically competent Xenopus before metamorphosis. J. Immunol. 1986, 137, 3891-3899. [PubMed]

12. Salter-Cid, L.; Nonaka, M.; Flajnik, M.F. Expression of MHC class Ia and class Ib during ontogeny: High expression in epithelia and coregulation of class Ia and lmp7 genes. J. Immunol. 1998, 160, $2853-2861$. [PubMed]

13. Rollins-Smith, L.A.; Flajnik, M.F.; Blair, P.J.; Davis, A.T.; Green, W.F. Involvement of thyroid hormones in the expression of MHC class I antigens during ontogeny in Xenopus. Dev. Immunol. 1997, 5, 133-144. [CrossRef] [PubMed]

14. AmphibiaWeb. Available online: https://amphibiaweb.org (accessed on 2 February 2019).

15. Feng, Y.-J.; Blackburn, D.C.; Liang, D.; Hillis, D.M.; Wake, D.B.; Cannatella, D.C.; Zhang, P. Phylogenomics reveals rapid, simultaneous diversification of three major clades of Gondwanan frogs at the Cretaceous-Paleogene boundary. Proc. Natl. Acad. Sci. USA 2017, 114, E5864-E5870. [CrossRef]

16. Igawa, T.; Kurabayashi, A.; Usuki, C.; Fujii, T.; Sumida, M. Complete mitochondrial genomes of three neobatrachian anurans: A case study of divergence time estimation using different data and calibration settings. Gene 2008, 407, 116-129. [CrossRef]

17. Didinger, C.; Eimes, J.A.; Lillie, M.; Waldman, B. Multiple major histocompatibility complex class I genes in asian anurans: Ontogeny and phylogeny of expression. Dev. Comp. Immunol. 2016, 70, 69-79. [CrossRef] 
18. Lau, Q.; Igawa, T.; Minei, R.; Kosch, T.A.; Satta, Y. Transcriptome analyses of immune tissues from three Japanese frogs (genus Rana) reveals their utility in characterizing major histocompatibility complex class II. BMC Genom. 2017, 18, 994. [CrossRef]

19. Tahara, Y. Table of the normal developmental stages of the frog, Rana japonica. 1. Early development (Stages 1-25). Jpn. J. Exp. Morphol. 1959, 13, 49-60.

20. Tahara, Y. Table of normal developmental stages of the frog, Rana japonica. 2. Late development (stages 26-40). Mem. Osaka Kyoiku Univ. (Nat. Sci. Appl. Sci.) 1974, 23, 33-53.

21. Lau, Q.; Igawa, T.; Komaki, S.; Satta, Y. Characterisation of major histocompatibility complex class I genes in Japanese Ranidae frogs. Immunogenetics 2016, 68, 797-806. [CrossRef]

22. Lau, Q.; Igawa, T.; Kosch, T.A.; Satta, Y. Selective constraint acting on TLR2 and TLR4 genes of Japanese Rana frogs. PeerJ 2018, 6, e4842. [CrossRef] [PubMed]

23. Nieuwkoop, P.D.; Faber, J. Normal Table of Xenopus laevis (Daudin): A Systematical and Chronological Survey of the Development from the Fertilized Egg till the End of Metamorphosis; Garland Publishing, Inc.: New York, NY, USA, 1994; ISBN 0815318960.

24. Grabherr, M.G.; Haas, B.J.; Yassour, M.; Levin, J.Z.; Thompson, D.A.; Amit, I.; Adiconis, X.; Fan, L.; Raychowdhury, R.; Zeng, Q.; et al. Trinity: Reconstructing a full-length transcriptome without a genome from RNA-Seq data. Nat. Biotechnol. 2013, 29, 644-652. [CrossRef] [PubMed]

25. Bray, N.L.; Pimentel, H.; Melsted, P.; Pachter, L. Near-optimal probabilistic RNA-seq quantification. Nat. Biotechnol. 2016, 34, 525-527. [CrossRef] [PubMed]

26. Robinson, M.D.; McCarthy, D.J.; Smyth, G.K. edgeR: A Bioconductor package for differential expression analysis of digital gene expression data. Bioinformatics 2009, 26, 139-140. [CrossRef] [PubMed]

27. James-Zorn, C.; Ponferrada, V.; Fisher, M.E.; Burns, K.; Fortriede, J.; Segerdell, E.; Karimi, K.; Lotay, V.; Wang, D.Z.; Chu, S.; et al. Navigating xenbase: An integrated Xenopus genomics and gene expression database. Methods Mol. Biol. 2018, 1757, 251-305. [CrossRef]

28. Huang, D.W.; Sherman, B.T.; Lempicki, R.A. Systematic and integrative analysis of large gene lists using DAVID bioinformatics resources. Nat. Protoc. 2009, 4, 44-57. [CrossRef] [PubMed]

29. McDiarmid, R.W.; Altig, R. Research: materials and techniques. In Tadpoles: The Biology of Anuran Larvae; McDiarmid, R.W., Altig, R., Eds.; University of Chicago Press: Chicago, IL, USA, 1999; pp. 7-23.

30. Gosner, K.L. A simplified table for staging anuran embryos larvae with notes on identification. Herpetologica 1960, 16, 183-190.

31. Flajnik, M.F.; Ohta, Y.; Greenberg, A.S.; Salter-Cid, L.; Carrizosa, A.; Du Pasquier, L.; Kasahara, M. Two ancient allelic lineages at the single classical class I locus in the Xenopus MHC. J. Immunol. 1999, 163, 3826-3833.

(C) 2020 by the authors. Licensee MDPI, Basel, Switzerland. This article is an open access article distributed under the terms and conditions of the Creative Commons Attribution (CC BY) license (http://creativecommons.org/licenses/by/4.0/). 